\title{
7. MATERNAL COUNTRY OF BIRTH
}

In this section maternal countries of birth are combined into English-speaking and other regional groups. The country groups and individual countries are listed in Appendix 3. Recent trends in confinements for individual maternal countries of birth are shown in Table 4 (page 20).

\section{Trends in confinements}

Between 2000 and 2004, about 20 per cent of mothers were born in non-English speaking countries (Table 54). Over the 5 year period, there were slight declines in the percentage of mothers born in Southern Europe, South East Asia and North East Asia, and slight increases in the percentage of mothers born in Middle East and Africa, and Southern Asia.

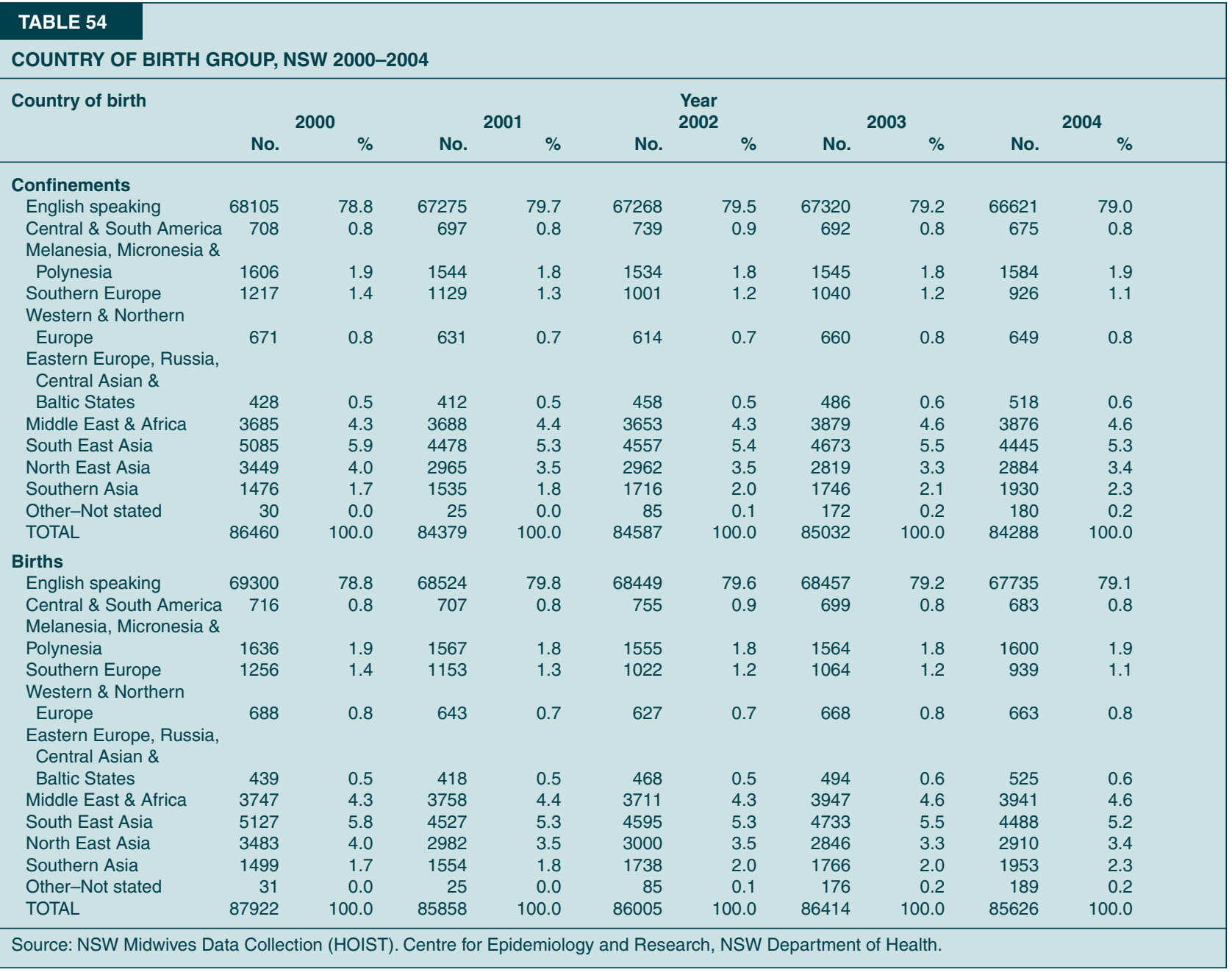




\section{Maternal age}

Births to teenage mothers were more common among mothers born in English-speaking countries than nonEnglish speaking countries (Table 55, Figure 4), while the largest proportions of mothers aged 35 years and over were among mothers born in Western and Northern Europe (36.7 per cent) and Southern Europe (31.3 per cent).

\begin{tabular}{|c|c|c|c|c|c|c|c|c|c|c|}
\hline \multirow[t]{3}{*}{ Country of birth group } & \multicolumn{10}{|c|}{ Maternal age (years) } \\
\hline & \multicolumn{2}{|c|}{$12-19$} & \multicolumn{2}{|c|}{$20-34$} & \multicolumn{2}{|c|}{$35+$} & \multicolumn{2}{|c|}{ Not stated } & \multicolumn{2}{|c|}{ TOTAL } \\
\hline & No. & $\%$ & No. & $\%$ & No. & $\%$ & No. & $\%$ & No. & $\%$ \\
\hline English speaking & 3080 & 4.6 & 51005 & 76.6 & 12522 & 18.8 & 14 & 0.0 & 66621 & 100.0 \\
\hline Central \& South America & 9 & 1.3 & 474 & 70.2 & 191 & 28.3 & 1 & 0.1 & 675 & 100.0 \\
\hline Melanesia, Micronesia \& Polynesia & 36 & 2.3 & 1163 & 73.4 & 385 & 24.3 & 0 & 0.0 & 1584 & 100.0 \\
\hline Southern Europe & 13 & 1.4 & 622 & 67.2 & 290 & 31.3 & 1 & 0.1 & 926 & 100.0 \\
\hline Western \& Northern Europe & 5 & 0.8 & 405 & 62.4 & 238 & 36.7 & 1 & 0.2 & 649 & 100.0 \\
\hline \multicolumn{11}{|l|}{ Eastern Europe, Russia, Central } \\
\hline Asian \& Baltic States & 2 & 0.4 & 408 & 78.8 & 108 & 20.8 & 0 & 0.0 & 518 & 100.0 \\
\hline Middle East \& Africa & 143 & 3.7 & 2967 & 76.5 & 766 & 19.8 & 0 & 0.0 & 3876 & 100.0 \\
\hline South East Asia & 58 & 1.3 & 3353 & 75.4 & 1034 & 23.3 & 0 & 0.0 & 4445 & 100.0 \\
\hline North East Asia & 18 & 0.6 & 1971 & 68.3 & 895 & 31.0 & 0 & 0.0 & 2884 & 100.0 \\
\hline Southern Asia & 18 & 0.9 & 1617 & 83.8 & 295 & 15.3 & 0 & 0.0 & 1930 & 100.0 \\
\hline Other-Not stated & 5 & 2.8 & 129 & 71.7 & 45 & 25.0 & 1 & 0.6 & 180 & 100.0 \\
\hline TOTAL & 3387 & 4.0 & 64114 & 76.1 & 16769 & 19.9 & 18 & 0.0 & 84288 & 100.0 \\
\hline
\end{tabular}

\section{FIGURE 4}

MATERNAL AGE BY COUNTRY OF BIRTH GROUP, NSW 2004

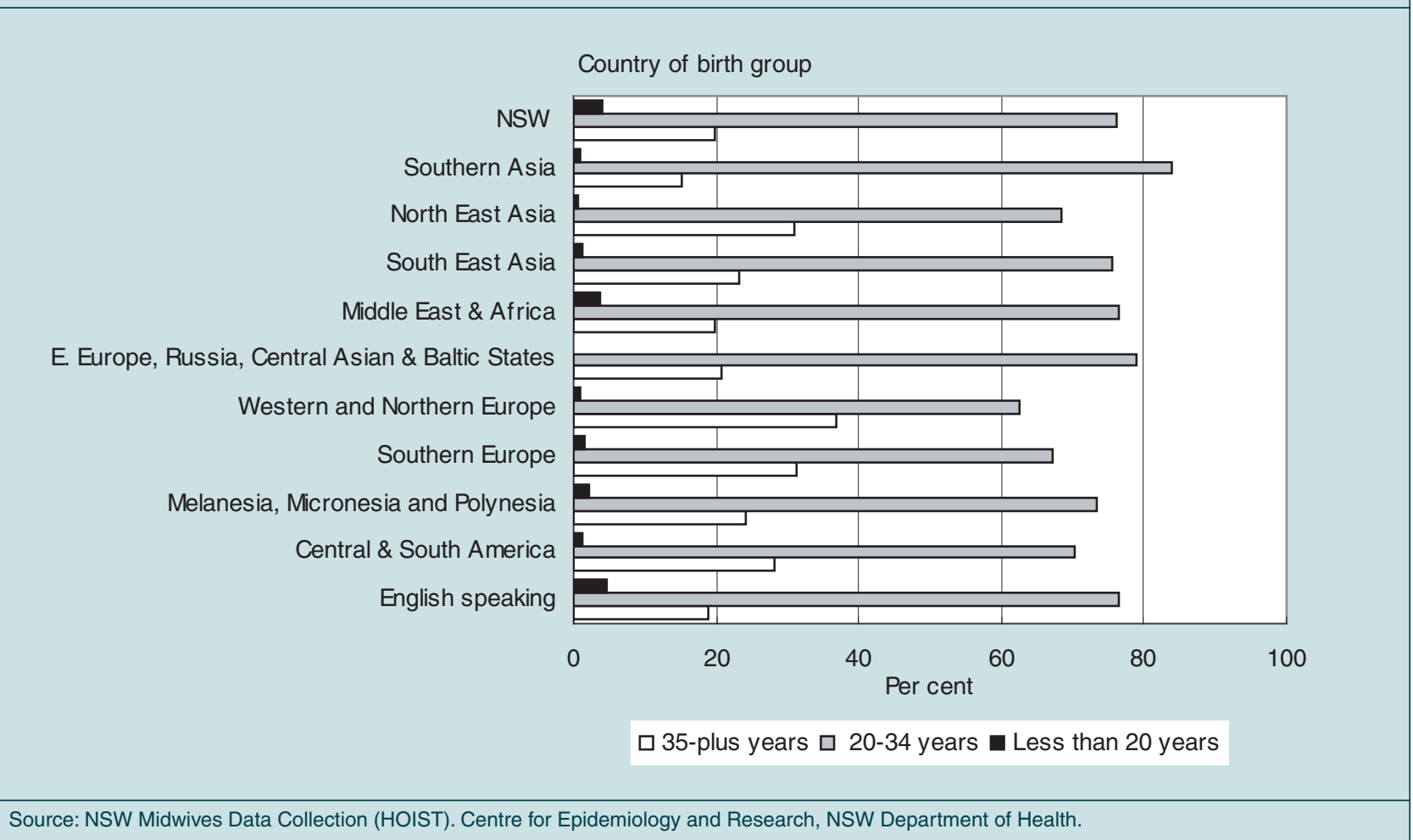




\section{Health area of residence}

In 2004, the proportion of mothers born in non-English speaking countries was highest in the Sydney South West Area (38.6 per cent), followed by the Sydney West Area (29.1 per cent).

Five per cent of mothers were born in South East Asian countries, 53.9 per cent of whom were resident in the
Sydney South West Area. Almost 5 per cent of mothers were born in Middle Eastern or African countries and 80.3 per cent of these mothers were resident in the Sydney South West or Sydney West Areas. A further 3.4 per cent of mothers were born in North East Asian countries and 2.3 per cent in Southern Asian countries, with the majority living in metropolitan areas (Table 56).

\section{TABLE 56}

HEALTH AREA OF RESIDENCE BY MATERNAL COUNTRY OF BIRTH GROUP, NSW 2004"

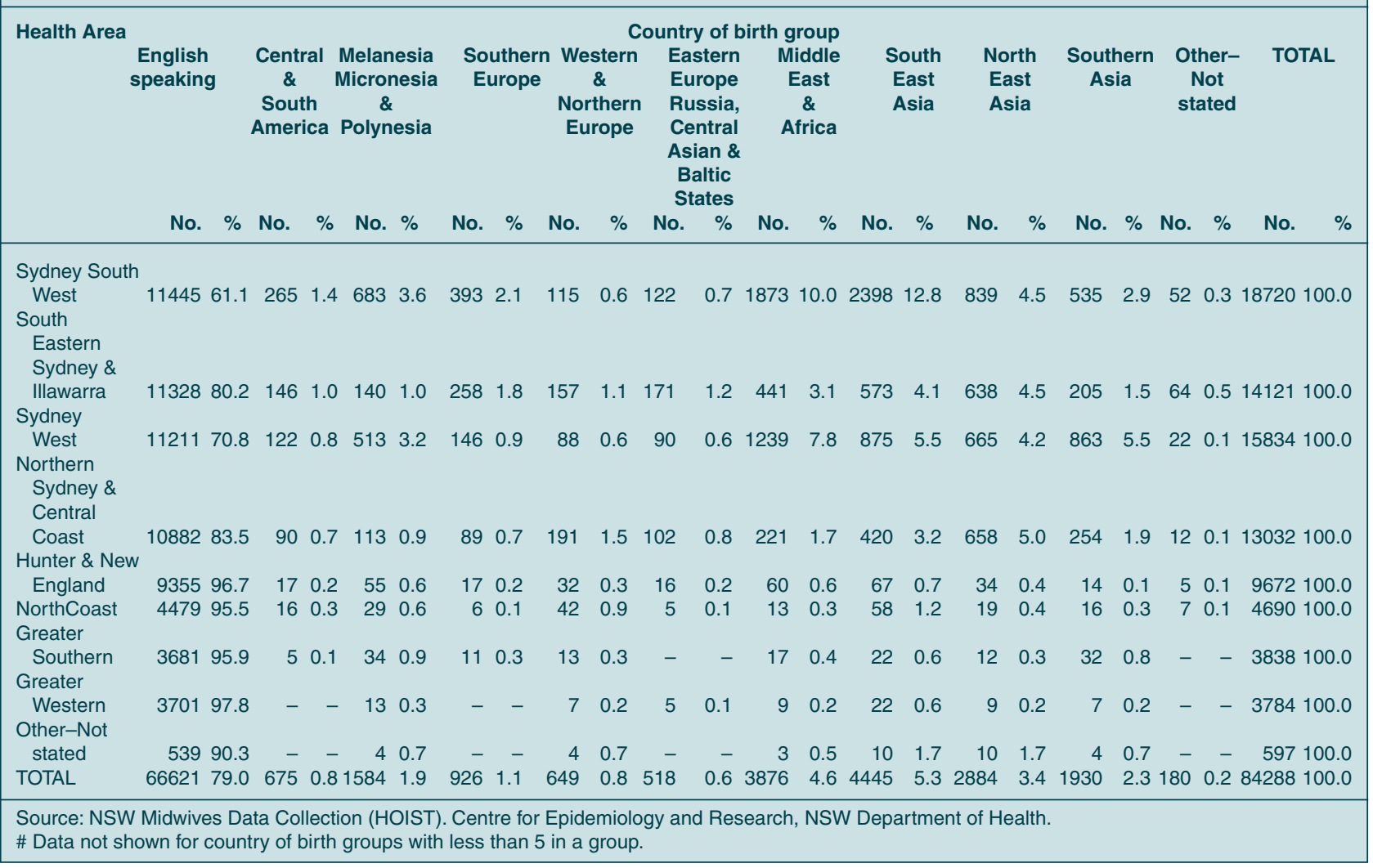




\section{Booking status}

In 2004, 97.3 per cent of all mothers were booked at the hospital of birth. The lowest rate (94.7 per cent) was in mothers born in Melanesia, Micronesia and Polynesia. This compared with 97.3 per cent of mothers born in English speaking countries and over 97 per cent of mothers in other country of birth groups.

\section{Duration of pregnancy at first antenatal visit}

In $2004,87.5$ per cent of all mothers commenced antenatal care before 20 weeks gestation. There was some variation between country of birth groups, with 89.3 per cent of mothers born in English speaking countries commencing antenatal care before 20 weeks gestation, compared with 64.2 per cent of mothers born in Melanesia, Micronesia, and Polynesia, and 75.3 per cent of mothers born in the Middle East and Africa (Table 57).

\section{TABLE 57}

\section{DURATION OF PREGNANCY AT FIRST ANTENATAL VISIT BY COUNTRY OF BIRTH GROUP, NSW 2004}

\begin{tabular}{|c|c|c|c|c|c|c|c|c|}
\hline \multirow[t]{3}{*}{ Country of birth group } & \multicolumn{8}{|c|}{ Duration of pregnancy at first antenatal visit (weeks) } \\
\hline & \multicolumn{2}{|c|}{$0-19$} & \multicolumn{2}{|c|}{$20+$} & \multicolumn{2}{|c|}{ Not stated } & \multicolumn{2}{|c|}{ TOTAL } \\
\hline & No. & $\%$ & No. & $\%$ & No. & $\%$ & No. & $\%$ \\
\hline English speaking & 59494 & 89.3 & 6625 & 9.9 & 502 & 0.8 & 66621 & 100.0 \\
\hline Central \& South America & 597 & 88.4 & 78 & 11.6 & 0 & 0.0 & 675 & 100.0 \\
\hline Melanesia, Micronesia \& Polynesia & 1017 & 64.2 & 539 & 34.0 & 28 & 1.8 & 1584 & 100.0 \\
\hline Southern Europe & 826 & 89.2 & 99 & 10.7 & 1 & 0.1 & 926 & 100.0 \\
\hline Western \& Northern Europe & 572 & 88.1 & 77 & 11.9 & 0 & 0.0 & 649 & 100.0 \\
\hline Eastern Europe, Russia, & & & & & & & & \\
\hline Central Asian \& Baltic States & 445 & 85.9 & 73 & 14.1 & 0 & 0.0 & 518 & 100.0 \\
\hline Middle East \& Africa & 2917 & 75.3 & 946 & 24.4 & 13 & 0.3 & 3876 & 100.0 \\
\hline South East Asia & 3726 & 83.8 & 703 & 15.8 & 16 & 0.4 & 4445 & 100.0 \\
\hline North East Asia & 2403 & 83.3 & 478 & 16.6 & 3 & 0.1 & 2884 & 100.0 \\
\hline Southern Asia & 1641 & 85.0 & 285 & 14.8 & 4 & 0.2 & 1930 & 100.0 \\
\hline Other-Not stated & 137 & 76.1 & 31 & 17.2 & 12 & 6.7 & 180 & 100.0 \\
\hline TOTAL & 73775 & 87.5 & 9934 & 11.8 & 579 & 0.7 & 84288 & 100.0 \\
\hline
\end{tabular}

\section{Smoking in pregnancy}

In 2004, smoking at any time during pregnancy was more common among mothers born in English speaking countries than mothers born in non-English speaking countries (Table 58). About one in 6 mothers born in English speaking countries smoked at some time during pregnancy, compared to one in 10 or fewer mothers born in other country of birth groups.
Smoking in the second half of pregnancy poses the greatest risk to the health of both mother and baby. Four per cent of mothers who smoked during pregnancy quit before the second half of pregnancy. Of mothers who did smoke in the second half of pregnancy, mothers born in English speaking countries were more likely to smoke more than 10 cigarettes per day compared to mothers born in other country of birth groups (Table 59).

\section{TABLE 58}

SMOKING IN PREGNANCY BY COUNTRY OF BIRTH GROUP, NSW 2004

\begin{tabular}{|c|c|c|c|c|c|c|c|c|}
\hline \multirow[t]{3}{*}{ Country of birth group } & \multicolumn{8}{|c|}{ Smoking in pregnancy } \\
\hline & \multicolumn{2}{|c|}{ No } & \multicolumn{2}{|c|}{ Yes } & \multicolumn{2}{|c|}{ Not stated } & \multicolumn{2}{|c|}{ TOTAL } \\
\hline & No. & $\%$ & No. & $\%$ & No. & $\%$ & No. & $\%$ \\
\hline English speaking & 54842 & 82.3 & 11772 & 17.7 & 7 & 0.0 & 66621 & 100.0 \\
\hline Central \& South America & 648 & 96.0 & 27 & 4.0 & 0 & 0.0 & 675 & 100.0 \\
\hline Melanesia, Micronesia \& Polynesia & 1435 & 90.6 & 149 & 9.4 & 0 & 0.0 & 1584 & 100.0 \\
\hline Southern Europe & 824 & 89.0 & 102 & 11.0 & 0 & 0.0 & 926 & 100.0 \\
\hline Western \& Northern Europe & 609 & 93.8 & 40 & 6.2 & 0 & 0.0 & 649 & 100.0 \\
\hline \multicolumn{9}{|l|}{ Eastern Europe, Russia, } \\
\hline Central Asian \& Baltic States & 497 & 95.9 & 21 & 4.1 & 0 & 0.0 & 518 & 100.0 \\
\hline Middle East \& Africa & 3661 & 94.5 & 215 & 5.5 & 0 & 0.0 & 3876 & 100.0 \\
\hline South East Asia & 4361 & 98.1 & 84 & 1.9 & 0 & 0.0 & 4445 & 100.0 \\
\hline North East Asia & 2855 & 99.0 & 29 & 1.0 & 0 & 0.0 & 2884 & 100.0 \\
\hline Southern Asia & 1920 & 99.5 & 10 & 0.5 & 0 & 0.0 & 1930 & 100.0 \\
\hline Other-Not stated & 156 & 86.7 & 23 & 12.8 & 1 & 0.6 & 180 & 100.0 \\
\hline TOTAL & 71808 & 85.2 & 12472 & 14.8 & 8 & 0.0 & 84288 & 100.0 \\
\hline
\end{tabular}




\section{TABLE 59}

MOTHERS WHO SMOKED AT ALL DURING PREGNANCY BY NUMBER OF CIGARETTES SMOKED IN THE SECOND HALF OF PREGNANCY AND COUNTRY OF BIRTH GROUP, NSW 2004

\begin{tabular}{|c|c|c|c|c|c|c|c|c|c|c|c|c|}
\hline \multirow[t]{3}{*}{ Country of birth group } & \multicolumn{12}{|c|}{ Cigarettes smoked in the second half of pregnancy } \\
\hline & \multicolumn{2}{|c|}{ None } & \multicolumn{2}{|c|}{$\begin{array}{c}1-10 \\
\text { per day }\end{array}$} & \multicolumn{2}{|c|}{$\begin{array}{l}\text { More than } \\
10 \text { per day }\end{array}$} & \multicolumn{2}{|c|}{$\begin{array}{c}\text { Smoked, amount } \\
\text { not stated }\end{array}$} & \multicolumn{2}{|c|}{ Not stated } & \multicolumn{2}{|c|}{ TOTAL } \\
\hline & No. & $\%$ & No. & $\%$ & No. & $\%$ & No. & $\%$ & No. & $\%$ & No. & $\%$ \\
\hline English speaking & 439 & 3.7 & 5900 & 50.1 & 5150 & 43.7 & 275 & 2.3 & 8 & 0.1 & 11772 & 100.0 \\
\hline Central \& South America & 3 & 11.1 & 17 & 63.0 & 5 & 18.5 & 1 & 3.7 & 1 & 3.7 & 27 & 100.0 \\
\hline Melanesia, Micronesia \& Polynesia & 11 & 7.4 & 91 & 61.1 & 44 & 29.5 & 3 & 2.0 & 0 & 0.0 & 149 & 100.0 \\
\hline Southern Europe & 3 & 2.9 & 57 & 55.9 & 41 & 40.2 & 1 & 1.0 & 0 & 0.0 & 102 & 100.0 \\
\hline Western \& Northern Europe & 4 & 10.0 & 20 & 50.0 & 15 & 37.5 & 1 & 2.5 & 0 & 0.0 & 40 & 100.0 \\
\hline \multicolumn{13}{|l|}{ Eastern Europe, Russia, Central } \\
\hline Asian \& Baltic States & 1 & 4.8 & 12 & 57.1 & 6 & 28.6 & 2 & 9.5 & 0 & 0.0 & 21 & 100.0 \\
\hline Middle East \& Africa & 10 & 4.7 & 117 & 54.4 & 77 & 35.8 & 11 & 5.1 & 0 & 0.0 & 215 & 100.0 \\
\hline South East Asia & 6 & 7.1 & 51 & 60.7 & 25 & 29.8 & 2 & 2.4 & 0 & 0.0 & 84 & 100.0 \\
\hline North East Asia & 5 & 17.2 & 20 & 69.0 & 4 & 13.8 & 0 & 0.0 & 0 & 0.0 & 29 & 100.0 \\
\hline Southern Asia & 2 & 20.0 & 5 & 50.0 & 2 & 20.0 & 1 & 10.0 & 0 & 0.0 & 10 & 100.0 \\
\hline Other-Not stated & 1 & 4.3 & 13 & 56.5 & 9 & 39.1 & 0 & 0.0 & 0 & 0.0 & 23 & 100.0 \\
\hline TOTAL & 485 & 3.9 & 6303 & 50.5 & 5378 & 43.1 & 297 & 2.4 & 9 & 0.1 & 12472 & 100.0 \\
\hline
\end{tabular}

\section{Medical conditions and obstetric complications}

In 2004, 1.5 per cent of mothers born in Melanesia, Micronesia, and Polynesia were reported to have diabetes mellitus, over twice the rate for all mothers in NSW, though the number of mothers is small (Table 60). The rates of gestational diabetes in mothers born in Asian countries and Melanesia, Micronesia, and Polynesia were at least twice the rate for all mothers in NSW.
Overall, 1.1 per cent of mothers were reported to have essential hypertension, and 5.5 per cent were reported to have pre-eclampsia. Rates of reported pre-eclampsia were lower among mothers born in North East Asian countries than other country of birth groups.

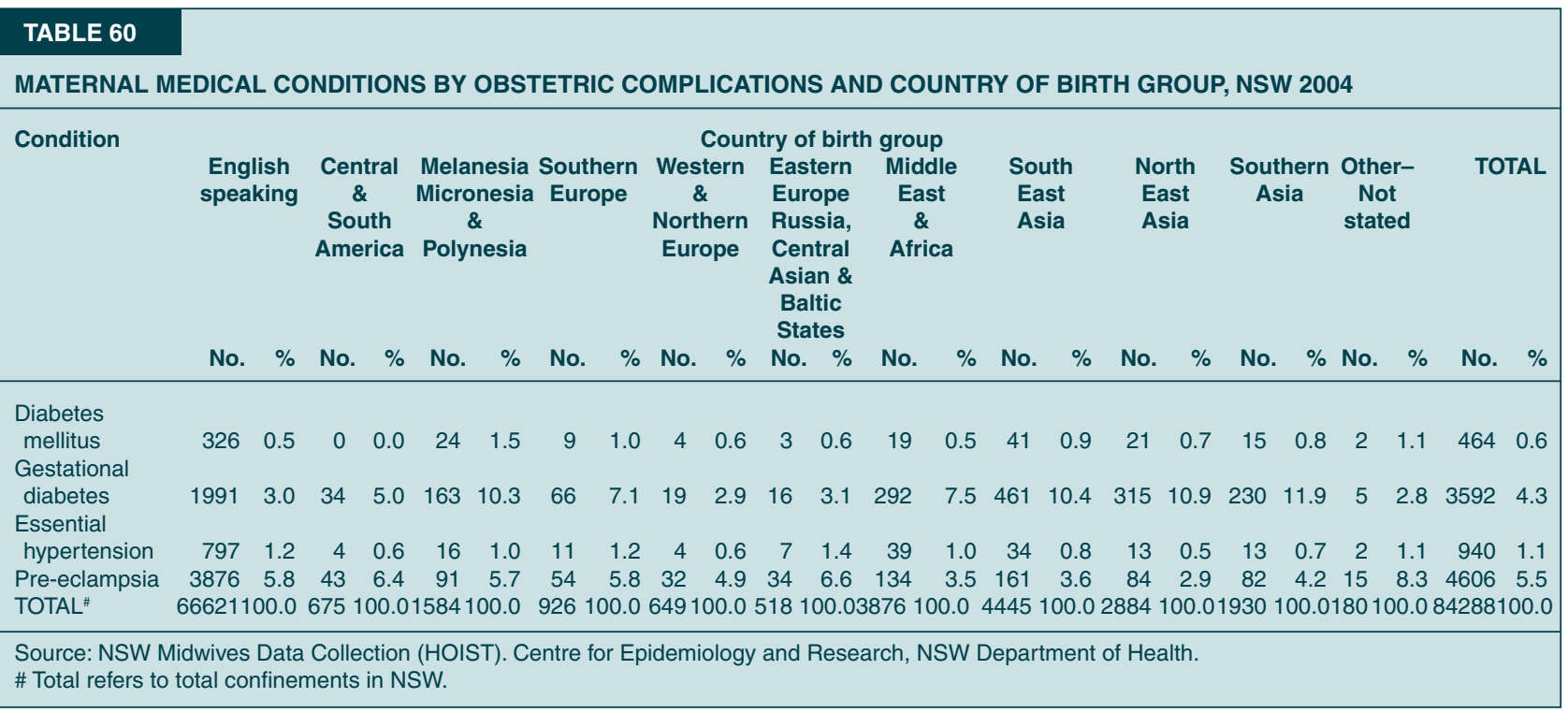




\section{Labour and delivery}

Mothers born in non-English speaking countries were more likely to have a spontaneous onset of labour than mothers born in English speaking countries, and were less likely to be induced (Table 61).

Mothers born in the Middle East and Africa and Melanesia, Micronesia and Polynesia were more likely to have a normal vaginal delivery than mothers in other country of birth groups (Table 62). The highest caesarean section rates were among mothers born in Southern Asia (30.7 per cent) and Central and South America (31.3 per cent).

\begin{tabular}{|c|c|c|c|c|c|c|c|c|c|c|}
\hline \multirow[t]{2}{*}{$\begin{array}{l}\text { Country of } \\
\text { birth group }\end{array}$} & \multicolumn{2}{|c|}{ Spontaneous } & \multicolumn{2}{|c|}{ No labour\# } & \multicolumn{2}{|c|}{$\begin{array}{l}\text { Onset of labour } \\
\text { Induced }\end{array}$} & \multicolumn{2}{|c|}{ Not stated } & \multicolumn{2}{|c|}{ TOTAL } \\
\hline & No. & $\%$ & No. & $\%$ & No. & $\%$ & No. & $\%$ & No. & $\%$ \\
\hline English speaking & 38900 & 58.4 & 10452 & 15.7 & 17268 & 25.9 & 1 & 0.0 & 66621 & 100.0 \\
\hline Central \& South America & 420 & 62.2 & 125 & 18.5 & 130 & 19.3 & 0 & 0.0 & 675 & 100.0 \\
\hline Melanesia, Micronesia \& Polynesia & 1064 & 67.2 & 191 & 12.1 & 329 & 20.8 & 0 & 0.0 & 1584 & 100.0 \\
\hline Southern Europe & 589 & 63.6 & 142 & 15.3 & 195 & 21.1 & 0 & 0.0 & 926 & 100.0 \\
\hline Western \& Northern Europe & 420 & 64.7 & 106 & 16.3 & 123 & 19.0 & 0 & 0.0 & 649 & 100.0 \\
\hline \multicolumn{11}{|l|}{ Eastern Europe, Russia, Central } \\
\hline Asian \& Baltic States & 349 & 67.4 & 70 & 13.5 & 99 & 19.1 & 0 & 0.0 & 518 & 100.0 \\
\hline Middle East \& Africa & 2590 & 66.8 & 486 & 12.5 & 800 & 20.6 & 0 & 0.0 & 3876 & 100.0 \\
\hline South East Asia & 3173 & 71.4 & 586 & 13.2 & 686 & 15.4 & 0 & 0.0 & 4445 & 100.0 \\
\hline North East Asia & 1962 & 68.0 & 430 & 14.9 & 492 & 17.1 & 0 & 0.0 & 2884 & 100.0 \\
\hline Southern Asia & 1235 & 64.0 & 290 & 15.0 & 405 & 21.0 & 0 & 0.0 & 1930 & 100.0 \\
\hline Other-Not stated & 105 & 58.3 & 52 & 28.9 & 23 & 12.8 & 0 & 0.0 & 180 & 100.0 \\
\hline TOTAL & 50807 & 60.3 & 12930 & 15.3 & 20550 & 24.4 & 1 & 0.0 & 84288 & 100.0 \\
\hline
\end{tabular}

Source: NSW Midwives Data Collection (HOIST). Centre for Epidemiology and Research, NSW Department of Health.

\# No labour indicates elective caesarean section.

\section{TABLE 62}

\section{TYPE OF DELIVERY BY COUNTRY OF BIRTH GROUP, NSW 2004}

\begin{tabular}{|c|c|c|c|c|c|c|c|c|c|c|c|c|c|c|c|c|}
\hline \multirow{3}{*}{$\begin{array}{l}\text { Country of } \\
\text { birth group }\end{array}$} & \multirow{2}{*}{\multicolumn{2}{|c|}{$\begin{array}{l}\text { Normal } \\
\text { vaginal }\end{array}$}} & \multirow{2}{*}{\multicolumn{2}{|c|}{ Forceps }} & \multirow{2}{*}{\multicolumn{2}{|c|}{$\begin{array}{l}\text { Vacuum } \\
\text { extraction }\end{array}$}} & \multirow{2}{*}{\multicolumn{2}{|c|}{$\begin{array}{l}\text { Type o } \\
\text { Vaginal } \\
\text { breech }\end{array}$}} & \multirow{2}{*}{\multicolumn{2}{|c|}{$\begin{array}{l}\text { Elective } \\
\text { caesarean } \\
\text { section }\end{array}$}} & \multirow{2}{*}{\multicolumn{2}{|c|}{$\begin{array}{c}\text { Emergency } \\
\text { caesarean } \\
\text { section" }^{*}\end{array}$}} & \multirow{2}{*}{\multicolumn{2}{|c|}{$\begin{array}{c}\text { Not } \\
\text { stated }\end{array}$}} & \multirow{2}{*}{\multicolumn{2}{|c|}{ TOTAL }} \\
\hline & & & & & & & & & & & & & & & & \\
\hline & No. & $\%$ & No. & $\%$ & No. & $\%$ & No. & $\%$ & No. & $\%$ & No. & $\%$ & No. & $\%$ & No. & $\%$ \\
\hline $\begin{array}{l}\text { English speaking } \\
\text { Central \& South }\end{array}$ & 41317 & 62.0 & 2162 & 3.2 & 4521 & 6.8 & 277 & 0.4 & 10452 & 15.7 & 7886 & 11.8 & 6 & 0.0 & 66621 & 100.0 \\
\hline $\begin{array}{l}\text { America } \\
\text { Melanesia, Micronesi }\end{array}$ & $\&^{390}$ & 57.8 & 16 & 2.4 & 54 & 8.0 & 4 & 0.6 & 125 & 18.5 & 86 & 12.7 & 0 & 0.0 & 675 & 100.0 \\
\hline Polynesia & 1091 & 68.9 & 38 & 2.4 & 77 & 4.9 & 8 & 0.5 & 191 & 12.1 & 179 & 11.3 & 0 & 0.0 & 1584 & 100.0 \\
\hline $\begin{array}{l}\text { Southern Europe } \\
\text { Western \& Northern }\end{array}$ & 584 & 63.1 & 26 & 2.8 & 73 & 7.9 & 3 & 0.3 & 142 & 15.3 & 98 & 10.6 & 0 & 0.0 & 926 & 100.0 \\
\hline $\begin{array}{l}\text { Europe } \\
\text { Eastern Europe, Russ } \\
\text { Central Asian \& }\end{array}$ & 374 & 57.6 & 28 & 4.3 & 65 & 10.0 & 2 & 0.3 & 106 & 16.3 & 74 & 11.4 & 0 & 0.0 & 649 & 100.0 \\
\hline Baltic States & 322 & 62.2 & 22 & 4.2 & 41 & 7.9 & 1 & 0.2 & 70 & 13.5 & 62 & 12.0 & 0 & 0.0 & 518 & 100.0 \\
\hline Middle East \& Africa & 2694 & 69.5 & 91 & 2.3 & 210 & 5.4 & 19 & 0.5 & 486 & 12.5 & 376 & 9.7 & 0 & 0.0 & 3876 & 100.0 \\
\hline South East Asia & 2784 & 62.6 & 130 & 2.9 & 390 & 8.8 & 16 & 0.4 & 586 & 13.2 & 538 & 12.1 & 1 & 0.0 & 4445 & 100.0 \\
\hline North East Asia & 1651 & 57.2 & 142 & 4.9 & 291 & 10.1 & 7 & 0.2 & 430 & 14.9 & 363 & 12.6 & 0 & 0.0 & 2884 & 100.0 \\
\hline Southern Asia & 1056 & 54.7 & 104 & 5.4 & 169 & 8.8 & 8 & 0.4 & 290 & 15.0 & 303 & 15.7 & 0 & 0.0 & 1930 & 100.0 \\
\hline Other-Not stated & 103 & 57.2 & 3 & 1.7 & 11 & 6.1 & 2 & 1.1 & 52 & 28.9 & 9 & 5.0 & 0 & 0.0 & 180 & 100.0 \\
\hline TOTAL & 52366 & 62.1 & 2762 & 3.3 & 5902 & 7.0 & $34 \overline{7}$ & 0.4 & 12930 & 15.3 & 9974 & 11.8 & 7 & 0.0 & 84288 & 100.0 \\
\hline
\end{tabular}

Source: NSW Midwives Data Collection (HOIST). Centre for Epidemiology and Research, NSW Department of Health.

\# Emergency caesarean section includes caesarean sections where the onset of labour was not stated. 


\section{Birth weight}

The rate of low birth weight (less than 2,500 grams) in 2004 was 6.4 per cent in NSW. The highest rates of low birth weight were in babies of mothers born in Southern Asian countries (8.0 per cent) (Table 63). Babies of mothers born in North East Asian countries were least likely to be low birth weight.

\section{TABLE 63}

\section{BIRTH WEIGHT BY MATERNAL COUNTRY OF BIRTH GROUP, NSW 2004}

\begin{tabular}{|c|c|c|c|c|c|c|c|c|}
\hline \multirow{3}{*}{$\begin{array}{l}\text { Country of } \\
\text { birth group }\end{array}$} & \multicolumn{8}{|c|}{ Birth weight (grams) } \\
\hline & \multicolumn{2}{|c|}{ Less than 2,500} & \multicolumn{2}{|c|}{$2,500+$} & \multicolumn{2}{|c|}{ Not stated } & \multicolumn{2}{|c|}{ TOTAL } \\
\hline & No. & $\%$ & No. & $\%$ & No. & $\%$ & No. & $\%$ \\
\hline English speaking & 4287 & 6.3 & 63411 & 93.6 & 37 & 0.1 & 67735 & 100.0 \\
\hline Central \& South America & 37 & 5.4 & 646 & 94.6 & 0 & 0.0 & 683 & 100.0 \\
\hline Melanesia, Micronesia \& Polynesia & 109 & 6.8 & 1489 & 93.1 & 2 & 0.1 & 1600 & 100.0 \\
\hline Southern Europe & 62 & 6.6 & 877 & 93.4 & 0 & 0.0 & 939 & 100.0 \\
\hline Western \& Northern Europe & 36 & 5.4 & 627 & 94.6 & 0 & 0.0 & 663 & 100.0 \\
\hline \multicolumn{9}{|l|}{ Eastern Europe, Russia, Central } \\
\hline Asian \& Baltic States & 31 & 5.9 & 494 & 94.1 & 0 & 0.0 & 525 & 100.0 \\
\hline Middle East \& Africa & 248 & 6.3 & 3690 & 93.6 & 3 & 0.1 & 3941 & 100.0 \\
\hline South East Asia & 307 & 6.8 & 4177 & 93.1 & 4 & 0.1 & 4488 & 100.0 \\
\hline North East Asia & 146 & 5.0 & 2764 & 95.0 & 0 & 0.0 & 2910 & 100.0 \\
\hline Southern Asia & 157 & 8.0 & 1795 & 91.9 & 1 & 0.1 & 1953 & 100.0 \\
\hline Other-Not stated & 37 & 19.6 & 150 & 79.4 & 2 & 1.1 & 189 & 100.0 \\
\hline TOTAL & 5457 & 6.4 & 80120 & 93.6 & 49 & 0.1 & 85626 & 100.0 \\
\hline
\end{tabular}

\section{Gestational age}

The rate of prematurity (less than 37 weeks gestation) in 2004 was 7.3 per cent in NSW. The highest rates of prematurity were in babies of mothers born in Melanesia, Micronesia and Polynesia (8.0 per cent). Babies of mothers born in Western and Northern Europe were least likely to be premature (Table 64).

\section{TABLE 64}

GESTATIONAL AGE BY MATERNAL COUNTRY OF BIRTH GROUP, NSW 2004

\begin{tabular}{|c|c|c|c|c|c|c|c|c|}
\hline \multirow{3}{*}{$\begin{array}{l}\text { Country of } \\
\text { birth group }\end{array}$} & \multicolumn{8}{|c|}{ Gestational age (weeks) } \\
\hline & \multicolumn{2}{|c|}{ Less than 37} & \multicolumn{2}{|c|}{$37+$} & \multicolumn{2}{|c|}{ Not stated } & \multicolumn{2}{|c|}{ TOTAL } \\
\hline & No. & $\%$ & No. & $\%$ & No. & $\%$ & No. & $\%$ \\
\hline English speaking & 5054 & 7.5 & 62678 & 92.5 & 3 & 0.0 & 67735 & 100.0 \\
\hline Central \& South America & 40 & 5.9 & 643 & 94.1 & 0 & 0.0 & 683 & 100.0 \\
\hline Melanesia, Micronesia \& Polynesia & 128 & 8.0 & 1472 & 92.0 & 0 & 0.0 & 1600 & 100.0 \\
\hline Southern Europe & 63 & 6.7 & 875 & 93.2 & 1 & 0.1 & 939 & 100.0 \\
\hline Western \& Northern Europe & 36 & 5.4 & 627 & 94.6 & 0 & 0.0 & 663 & 100.0 \\
\hline Eastern Europe, Russia, Central & & & & & & & & \\
\hline Asian \& Baltic States & 37 & 7.0 & 488 & 93.0 & 0 & 0.0 & 525 & 100.0 \\
\hline Middle East \& Africa & 262 & 6.6 & 3679 & 93.4 & 0 & 0.0 & 3941 & 100.0 \\
\hline South East Asia & 303 & 6.8 & 4185 & 93.2 & 0 & 0.0 & 4488 & 100.0 \\
\hline North East Asia & 160 & 5.5 & 2750 & 94.5 & 0 & 0.0 & 2910 & 100.0 \\
\hline Southern Asia & 124 & 6.3 & 1829 & 93.7 & 0 & 0.0 & 1953 & 100.0 \\
\hline Other/Not stated & 40 & 21.2 & 149 & 78.8 & 0 & 0.0 & 189 & 100.0 \\
\hline TOTAL & 6247 & 7.3 & 79375 & 92.7 & 4 & 0.0 & 85626 & 100.0 \\
\hline
\end{tabular}




\section{Apgar score}

In 2004, 2.1 per cent of all babies (including stillborn babies) had an Apgar score of 7 or less at 5 minutes and 1.1 per cent had a score of less than 4 (Table 65). The rate of Apgar scores of less than 7 was highest among babies of mothers born in Melanesia, Micronesia and Polynesia (3.0 per cent).

\begin{tabular}{|c|c|c|c|c|c|c|c|c|c|c|}
\hline \multirow[t]{2}{*}{$\begin{array}{l}\text { Country of } \\
\text { birth group }\end{array}$} & \multicolumn{2}{|c|}{$0-4$} & \multicolumn{2}{|c|}{$5-6$} & \multicolumn{2}{|c|}{$\begin{array}{c}\text { Apgar score } \\
7+\end{array}$} & \multicolumn{2}{|c|}{ Not stated } & \multicolumn{2}{|c|}{ TOTAL } \\
\hline & No. & $\%$ & No. & $\%$ & No. & $\%$ & No. & $\%$ & No. & $\%$ \\
\hline English speaking & 703 & 1.0 & 649 & 1.0 & 66209 & 97.7 & 174 & 0.3 & 67735 & 100.0 \\
\hline Central \& South America & 12 & 1.8 & 4 & 0.6 & 666 & 97.5 & 1 & 0.1 & 683 & 100.0 \\
\hline Melanesia, Micronesia \& Polynesia & 30 & 1.9 & 18 & 1.1 & 1547 & 96.7 & 5 & 0.3 & 1600 & 100.0 \\
\hline Southern Europe & 7 & 0.7 & 15 & 1.6 & 917 & 97.7 & 0 & 0.0 & 939 & 100.0 \\
\hline Western \& Northern Europe & 3 & 0.5 & 6 & 0.9 & 654 & 98.6 & 0 & 0.0 & 663 & 100.0 \\
\hline Eastern Europe, Russia, Central & & & & & & & & & & \\
\hline Asian \& Baltic States & 4 & 0.8 & 7 & 1.3 & 513 & 97.7 & 1 & 0.2 & 525 & 100.0 \\
\hline Middle East \& Africa & 55 & 1.4 & 34 & 0.9 & 3845 & 97.6 & 7 & 0.2 & 3941 & 100.0 \\
\hline South East Asia & 52 & 1.2 & 57 & 1.3 & 4370 & 97.4 & 9 & 0.2 & 4488 & 100.0 \\
\hline North East Asia & 23 & 0.8 & 24 & 0.8 & 2861 & 98.3 & 2 & 0.1 & 2910 & 100.0 \\
\hline Southern Asia & 25 & 1.3 & 26 & 1.3 & 1896 & 97.1 & 6 & 0.3 & 1953 & 100.0 \\
\hline Other-Not stated & 7 & 3.7 & 4 & 2.1 & 175 & 92.6 & 3 & 1.6 & 189 & 100.0 \\
\hline TOTAL & 921 & 1.1 & 844 & 1.0 & 83653 & 97.7 & 208 & 0.2 & 85626 & 100.0 \\
\hline
\end{tabular}

\section{Perinatal outcomes}

In 2004, 99 per cent of babies born in NSW and reported to the MDC were born alive and survived until discharge from the hospital of birth (Table 66). The majority of perinatal deaths occurred among babies of mothers born in English speaking countries ( $\mathrm{n}=594,76.8$ per cent). There were a further 44 deaths among babies of mothers born in South East Asian countries, and 42 deaths among babies of mothers born in the Middle East and Africa-comprising 5.7 and 5.4 per cent respectively of all perinatal deaths reported to the MDC.

\section{TABLE 66}

PERINATAL OUTCOMES BY COUNTRY OF BIRTH GROUP, NSW 2004\#

Country of

birth group

Liveborn

Perinatal outcome

Neonatal

Not stated

Total death

Perinatal

surviving

$\% \quad$ No.

No. $\%$ rate/1,000

$\begin{array}{lllllll}\text { No. } & \% & \text { No. } & \% & \text { No. } & \% & \text { No. }\end{array}$

English speaking

Central \& South America

$\begin{array}{llll}67138 & 99.1 & 437 & 0 .\end{array}$

$0.6 \quad 157 \quad 0.2$

0.2
0.7

$0.0 \quad 67735$

100.0

8.8

Melanesia, Micronesia \&

67198

$1579 \quad 98.7$

98.7
99.0

930

99.0

$\begin{array}{ll}16 & 1.0\end{array}$

1.0
0.7

5

Western \& Northern Europe

$\begin{array}{lll}663 & 100.0 & 0\end{array}$

0.0

$\begin{array}{ll}5 & 0.3 \\ 2 & 0.2\end{array}$

0.3
0.2

683

100.0

17.6

Eastern Europe, Russia,

Central Asian \&

Baltic States

Middle East \& Africa

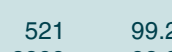

$\begin{array}{lll}99.2 & 3 & 0.6\end{array}$

$3899 \quad 98.9$

South East Asia

North East Asia

Southern Asia

Other-Not stated

$4444 \quad 99.0$

$\begin{array}{ll}2892 & 99.4 \\ 1931 & 98.9\end{array}$

1931
181

84849

95.8
99.1

$\begin{array}{rr}3 & 0.6 \\ 32 & 0.8 \\ 27 & 0.6 \\ 12 & 0.4 \\ 17 & 0.9 \\ 3 & 1.6 \\ 561 & 0.7\end{array}$

$\begin{array}{rr}0.6 & 1 \\ 0.8 & 10 \\ 0.6 & 17 \\ 0.4 & 6 \\ 0.9 & 5 \\ 1.6 & 4 \\ 0.7 & 212\end{array}$

$\begin{array}{rl}1 & 0.2 \\ 10 & 0.3 \\ 17 & 0.4 \\ 6 & 0.2 \\ 5 & 0.3 \\ 4 & 2.1 \\ 212 & 0.2\end{array}$

0.2

0.3

0.4

0.3

2.1
0.2

0

$\begin{array}{lll}0.0 & 1600 \quad 100.0\end{array}$

13.1

9.6

Source: NSW Midwives Data Collection (HOIST). Centre for Epidemiology and Research, Department of Health.

\# Perinatal deaths include deaths reported to the MDC only. As the MDC form is completed at discharge or transfer of the baby, deaths occurring after this time may not be reported to the MDC. 